\title{
Introduction to Virtual Reality Technology Application in Medical Education
}

\author{
Yinan Yang ${ }^{\mathrm{a}}$ \\ Department of Computer Science, North China Electric Power University, Baoding 071000, China \\ a2754869937@qq.com
}

Keywords: Virtual reality technology, education, medical application, virtual operation.

\begin{abstract}
Since the theory of virtual reality unfolded before our eyes, computer technology and interaction technology develops. Virtual reality technology gradually applied to a large amount of field, and brought its remarkable results. As an integral part of human life, medical education is drawn people's attention extensively. In this paper, we describe the concept, characteristics of virtual reality technology and analyze the applications of virtual reality technology in medical education. At last, we also stress the application expectation in virtual reality technology in the field of medical education.
\end{abstract}

\section{Introduction}

The development of science and technology are more and more quick in recent years, the things that most people imagine become reality, virtual reality technology emerges into the front of people, and its mysterious veil is unveiled. Virtual reality technology as one of the important means for promoting the development of the medicine, which has been received the attention of the domestic and foreign numerous scientific research personnel. Recently, the domestic and foreign research and practice of virtual reality technology has made remarkable achievements, and how to apply the technology further into the field of medicine, which has become a topic that relevant researchers commonly concern. This article from the aspect of medical education, the applications of virtual reality technology in classroom teaching and clinical teaching are analyzed in detail.

\section{Summary of Virtual Reality Technology}

\subsection{Concept of Virtual Reality Technology}

VR (Virtual Reality, VR), which is also named artificial environment, vision technology, VR create a 3D virtual world by using the computer simulation technology, which offers users various senses simulation on vision, hearing, smell, touch, it let users immersive, and can timely and unlimited to observe things in the three-dimensional space. When uses moving, the computer can carry out corresponding complex operation immediately, it will be transmitted precise three-dimensional video to the user and produce a telepresence. Virtual reality technology is integrated with computer graphics, computer simulation, artificial intelligence, sensor, display, and network parallel processing and other technologies results latest development achievements, which is a kind of high-tech simulation system aided generated by computer technology.

\subsection{Basic features of virtual reality technology [1]}

The main feature of VR technology is: Multi-Sensory, immersion, Interactivity, Imagination. These features enable users enter into an interactive 3D virtual space environment generated by the computer simulation, interact and communicate with it. Through the interaction between the user and the simulation environment, and with the help of perception and cognition ability that men themselves touch things, it can enlighten the minds of users, and help users all-round obtain all kinds of space information and logical information that environment contains.

\subsubsection{Multi-sensory}

Multi-sensory is besides having the visual perception that general computer technology have, which also has a auditory perception, tactile perception, force perception, motion perception, and even contains scent and taste perception, etc. Ideal virtual reality technology should have awareness 
that all mankind can have. Due to the related technology, especially the limitation of sensing technology, the perception function that the virtual reality technology have are limited to visual perception, auditory perception, tactile perception, force perception, motion perception these kinds.

\subsection{Immersion}

Immersion sense is also called telepresence, which is the real extent that user feel as a leading role in simulating environment. Ideal simulation environment should be able to make users difficult to distinguish true and false, enabling users to fully into the 3D virtual environment that computer create, everything seems true in this environment, it sounds really, it move really, even smell, taste and other perceptions are true, as we feel the same as in the real world.

\subsubsection{Interactivity}

Interaction is the operational extent that user on object in the simulation environment and feedback natural degree (including real time) from the environment. For example, users can directly use hands to grab the virtual objects that exist in the simulation environment, hands feel hold things at this moment, and can feel the weight of the object, the caught objects in the field of vision can move as the hands move immediately.

\subsubsection{Imagination}

Imagination emphasize the virtual reality technology have a broad imaginational space, which can broaden the human cognitive scope, it not only can reproduce the real environment, but also construct objective that does not exist, even environment may not happen. The real imagination of time and space environment, (namely inspiration thinking, the process of obtaining information) is the ultimate goal of virtual reality technology.

Because the first letter of immersion, interactivity and imagination three features English word is I, so the three features are also usually named 3I feature.

Generally speaking, a complete virtual reality system is constituted by the virtual environment, virtual environment processor that take high performance computer as the core, visual system that take head-mounted displays as the core, auditory system that take speech recognition, voice synthesis and positioning as the core, position attitude tracking equipment that take direction tracker, data gloves and clothing as the main body, as well as taste, smell, touch and force feedback system function units.

\section{Application of Virtual Reality Technology in The Field of Medical Education}

With the continuous development of modern medical technology, science and technology, virtual reality technology has been widely applied in the field of medical classroom teaching and clinical teaching.

\subsection{Virtual Human}

The virtual human body is digitized body structure, through the analysis of the computer technology and image processing technology, a seemingly real simulated human body will appear in computer screen, then the research achievements of human body function are further digitized, information scientists turn it into computer sign language, and impose the human body form framework. Through the intersection and integration of virtual reality technology, and the regulation of operator, this "virtual person" will be able to imitate all kinds of reaction that real person make, if set voice and force feedback device, it also can provide visual, hearing, touch and other intuitive and natural real-time sense. [2]

Traditional medical teaching mode are taught by the way such as PPT interpretation, specimen video by teachers, students are difficult to from intuitive feelings on stereoscopic structure from the aspect of human body, only observe in the experimental course through the specimen model structure and strengthen the memory. But the specimen model after numerous students' repeated uses, which is easy to damage, and the body specimen is increasingly scarce, which makes the opportunities that the students observe the human body model to the nearest are reduced year by year, it is unable to meet the needs of medical teaching. This requires people to find a new teaching tool as soon as possible, 
help students intuitively and profoundly understand the human body, and do not need to consume more teaching resources.

In 1989, the U.S. national library of medicine (while NLM) put forward an idea, hope to establish a medical image library and provide images retrieval system of biomedical literature, then the health sciences center of university of Colorado undertakes acquisition work of human body section image, and this project are named Visible Human Project (VHP). This project was obtained a man and a woman two groups including CT, MRI and slice image data sets from 1994 to 1996. [2] This slice image data sets issued by VHP is widely used in the world, spacing of male section is $1.0 \mathrm{~mm}$ and has 1878 cross section; spacing of female section is $0.33 \mathrm{~mm}$ and has 5190 section. At present, this visible man database has become ideal basis that constructs electronic medical library and virtual anatomic environment.

Germany University Hamburg medical mathematics and computer institute do anatomy 3D virtual visualization to study virtual human man atlas (VOXEL - MAN), subject's CT and the MRT cross-sectional images or histology section is established to the space model, artificial intelligence technology is used to designed the each human body part into the different areas of knowledge into the space model. Learners can freely operate in 3D human body space and corresponding text information. [3]

The 3D human body image generated by computer aided can make the students from all-round, multi-angle; deeply observe internal structures of human organs and produce direct and profound impression, which greatly improves the learning efficiency.

\subsection{Virtual Surgery System}

Virtual surgery system is one of the typical applications of virtual reality technology in the medical field. It start from medical image data, reconstruct the virtual human tissue model by using computer graphics, and simulate the virtual medical environment, using tactile interaction device and interactive operation system. Virtual surgery system provides medical workers with a virtual 3D space environment as well as the interactive operation platform, which can realistically simulate the whole process of clinical surgery. Compared with traditional surgery teaching, virtual surgery has non-damage, repeatability, nature-originated, etc.

Virtual surgery system can solve the operation error problem caused by unskilled operation, it can be repeatedly simulate and restore the real surgery scene and process, students are familiar with the operation steps in the process of practice, reduced the expensive experiments on animals at the same time, the teaching cost are reduced and the education resources are saved.

Through the virtual surgery system, users can complete from cutting, hemostasis, separation, lesions manipulation and wound closure every operation step, it also can timely analyze and evaluate the various steps and results in simulation process, and pointedly correct errors in the operation and experience possible unexpected situations in clinical surgery, it facilitates students to gather clinical skills and practical experience, and has repeatability, zero risk, low cost and other advantages.

In the use process of virtual surgery system, students can do familiar training with the aid of virtual environment, not only reduced the training cost, also improve the operation proficiency, reduce the errors in the actual operation process. Medical workers can also simulate operation situation through this system, repeated attempts to sophisticated operation, in order to perfect the surgical design step, improve surgical skills, reduce the risk of surgery for patients, and reduce the patients' pain after the operation.

At present, virtual surgery system are used into medical teaching, these examples has happened in the country. For example, Xiamen University launched "virtual liver surgery system" and "virtual" eye surgery system, doctors can input operation program into the system in advance, by using CT images, intuitively carry out surgery simulation, according to the plan and implement virtual surgery, compare and improve the different operation scheme, finally choose the highest degree safety and precision operation plan, improve the success rate of surgery.

\subsection{Virtual Laboratory}


Virtual laboratory is made up of virtual experiments that take computer simulation technology as the core and network structure that run the virtual experiment. Its core virtual experiment in accordance with the basic requirements of experimental teaching, virtual reality technology are used in the computer system to realize all kinds of virtual experiment environment, users can use a variety of virtual experiment instruments and equipment like in the real environment, the established experimental model are carried out real-time simulation operation, complete various predetermined experimental project, the effects of study or training effect are equivalent to or even better than the effect that obtained from operations in the real environment. [4]

Due to limited resources, limited equipment and other reasons, the teaching quality of medical colleges and universities are various, in recent years, virtual laboratory has been proved to be one of the effective methods to solve the problem.

Taking human anatomy in basic medical for example, the anatomy of the class as a basic course of medical courses, students must be repeated practice in the learning process, actually operate on the body, skilled in operation process and the human anatomy in order to have the actual hands-on ability, fully prepared to go to working posts. But due to the constant expansion of the colleges and universities in recent years, the number of students increased, but body resources that can be used for experiment teaching are limited, make students' teaching resources are increasingly scarce, using the virtual surgery system can solve this contradiction. Students can in virtual anatomy laboratory, personally dissect body anatomy on the virtual body, through the whole process of the anatomy and gather anatomical skills, observe human anatomy structure, obtain similar skills and experience the same as real-world anatomy experiment, so it makes up for lack of experience because the lack of experimental equipment and experimental item cause students' practical hands-on operation.

\section{Conclusion}

The virtual reality technology as an emerging subject, which is a product that many disciplines intersect and integrate, it involves the sensor, artificial intelligence, computer simulation technology, computer graphics and much other content. Virtual reality technology has huge development potential and broad prospects for development, but at the same time, we must clearly recognize that this emerging technology, there are many theoretical problems and technical barrier has been yet not conquer. Virtual reality technology must further strengthen the contact between users' organs and computers, and reduce its application costs, then play a more and more important role in the field of medical education. The potential advantages and broad prospects of virtual reality technology are consistently affirmative. We look forward to the day when virtual reality system can become powerful assistant of people's thinking creation and powerful tools that help people acquire new concept.

\section{References}

[1] Fan Lidong, Li Shuguang, Zhang Zhigang: The application of virtual reality technology in medical training, Journal of Traumatic Surgery, Vol. 10 (2008) No.6, p. 568-570.

[2] Zhong Shizhen: The present situation and prospects of digitized virtual human body research, Medical Journal of Chinese People's Liberation Army, Vol. 28 (2003) No.5, p.385-388.

[3] Zhang Han: The application of virtual reality technology in medical education (master's, Shandong Normal University, China), 2011, p. 3.

[4] Han Ying, Gao Xingya, Qi Xiao-hong, Zhu Guoqing, The establishment and application of physiological virtual laboratory, Medical Education, (2005) No.4, p. 63-65. 